\title{
Estudo numérico de escoamentos turbulentos com convecção forçada em cavidades cilíndricas: comparação entre LES e RANS
}

\author{
Numerical Study of Turbulence Modeling in Forced Convective Flows in Cylindrical Cavity: \\ comparison between LES and RANS
}

\author{
T. S. V. C. de Andrade ${ }^{1}$; M. P. Rodrigues ${ }^{1}$; L. A. Isoldi ${ }^{1}$; J. A. Souza ${ }^{1}$; F. R. \\ Centeno $^{2}$, L. A. O. Rocha ${ }^{3}$, F. H. R. França ${ }^{3}$, E. D. dos Santos ${ }^{1, *}$ \\ ${ }^{1}$ Escola de Engenharia, Universidade Federal do Rio Grande (FURG), 96021-900, Rio Grande-RS, Brasil. \\ ${ }^{2}$ Escola Politécnica, Universidade do Vale do Rio dos Sinos, 93022-000, São Leopoldo-RS, Brasil. \\ ${ }^{3}$ Departamento de Engenharia Mecânica, Universidade Federal do Rio Grande do Sul (UFRGS), 90050-170, Porto \\ Alegre - RS, Brasil. \\ *elizaldosantos@furg.br
}

(Recebido em 03 de setembro de 2014; aceito em 29 de dezembro de 2014)

O presente trabalho apresenta um estudo numérico comparando o emprego da Simulação de Grandes Escalas (LES) e Modelagem Clássica (RANS) com o modelo $k-\varepsilon$ para a predição dos campos médios e estatísticos em um escoamento turbulento com convecção forçada em cavidades cilíndricas (escoamento de base cisalhante livre) a $\operatorname{Re}_{\mathrm{D}}=22000$ e $\operatorname{Pr}=0.71$. No âmbito da convecção forçada, o principal propósito é verificar se o uso das diferentes metodologias para abordagem da turbulência pode conduzir a diferenças consideráveis nos fluxos convectivos nas superfícies da cavidade. Além disso, as diferenças dos campos médios e estatísticos de temperatura também são importantes para futuras análises de escoamentos com mecanismos combinados de convecção e radiação térmica em meios participantes, i.e., investigando a influência das Interações Turbulência-Radiação (TRI). As equações de conservação são resolvidas através do Método de Volumes Finitos (FVM). Os resultados revelaram que o jato apresentou uma maior penetração na cavidade cilíndrica para a simulação realizada com LES, enquanto um maior espalhamento radial foi observado no caso com o modelo RANS $k-\varepsilon$. Apesar de apresentarem uma tendência similar, diferenças significativas foram observadas para os campos médios, estatísticos e fluxos convectivos preditos com LES e RANS.

Palavras-chave: LES, RANS, turbulência.

The present work presents a numerical study comparing the employment of Large Eddy Simulation (LES) and Reynolds Averaged Navier-Stokes (RANS) with $k-\varepsilon$ model for prediction of time-averaged and statistics fields for a forced convective turbulent flow in cylindrical cavities (free shear flow) at $\operatorname{Re}_{\mathrm{D}}=$ 22000 and $\operatorname{Pr}=0.71$. In the forced convective framework, the main purpose is to verify whether the use of different methodologies for turbulence approach can led to large differences in convective fluxes in the cavity surfaces. Moreover, the differences between the time-averaged fields of temperature and its statistics predicted with both methods are important for future analyses of turbulent flows with combined convective and radiative transfer in participant medium, i.e., investigating the Turbulence-Radiation Interactions (TRI). The conservation equations are solved by means of Finite Volume Method (FVM). Results revealed that the jet showed a higher penetration into the cylindrical cavity for simulation with LES, while a larger scattering in the radial direction is noticed for the simulations with RANS $k-\varepsilon$. In spite of the qualitatively similarity, it was noticed strongly differences between time-averaged, statistics and convective fluxes predicted with LES and RANS.

Keywords: LES, RANS, turbulence.

\section{INTRODUÇÃO}

Os escoamentos turbulentos estão presentes em inúmeras aplicações no âmbito da engenharia, como em aerodinâmica, turbomáquinas, equipamentos térmicos, processos de combustão, secções de exaustão de combustores, oceanografia e astrofísica. Além disso, sua 
caracterização representa um problema altamente desafiador pela elevada complexidade da fenomenologia física envolvida [1,2].

Com relação à abordagem numérica, as três principais metodologias para a solução dos escoamentos turbulentos são a Simulação Numérica Direta (DNS - do inglês: Direct Numerical Simulation), Simulação de Grandes Escalas (LES - do inglês: Large Eddy Simulation) e a Modelagem Clássica da Turbulência (RANS - do inglês: Reynolds Averaged Navier-Stokes) [3 $-6]$.

A metodologia DNS é a abordagem mais acurada e conduz a resultados comparáveis aos obtidos em experimentos em laboratório [3]. Essa metodologia consiste em resolver as equações de conservação de massa, quantidade de movimento e energia em todas as escalas do escoamento. Contudo, é necessário realizar discretizações (espacial e temporal) extremamente refinadas o que inviabiliza seu emprego para problemas práticos de engenharia [4].

A metodologia LES consiste em eliminar as pequenas escalas do escoamento (menores que uma determinada dimensão) através da utilização de um processo de filtragem espacial, modelando apenas as pequenas escalas que tem um comportamento mais isotrópico [4,5]. Essa metodologia permite estimar os campos instantâneos (velocidade, pressão e temperatura) para escoamentos com maiores números de Reynolds e dimensões mais próximas da realidade do que DNS. Em comparação com o RANS essa metodologia permite a obtenção de um comportamento mais universal e sem ajustes para os vários escoamentos de base como os cisalhantes livres e parietais. Entretanto, LES requer um tempo de processamento sensivelmente mais elevado do que os requeridos para soluções com RANS.

A modelagem clássica da turbulência (RANS) consiste na ideia de se realizar uma média temporal sobre as equações de conservação que modelam os escoamentos, sendo as variáveis do problema (velocidade, temperatura e pressão) decompostas em uma parcela média e outra flutuante no tempo [6]. Esses modelos são menos universais e precisam de ajustes para os diferentes escoamentos de base a serem simulados, possuindo dificuldades na abordagem de efeitos transientes como desprendimentos de vórtices, esteiras de vórtices e vórtices de KelvinHelmholtz em uma camada de mistura [6,7].

O emprego de RANS tem sido amplamente realizado na predição de diversos escoamentos internos em problemas práticos de engenharia, principalmente devido ao menor esforço computacional requerido por essa abordagem. Alguns estudos têm demonstrado que o emprego dessa metodologia conduz a predições satisfatórias de escoamentos internos parietais. Por exemplo, em Dos Santos et al. [8] os campos fluidodinâmicos e térmicos médios e estatísticos obtidos com LES e RANS foram bastante concordantes. Outros trabalhos focados na comparação entre as metodologias LES e RANS têm sido apresentados na literatura, Colleoni et al. [9] apresentaram uma comparação da troca térmica em um escoamento turbulento em placa solar. Neste estudo foi observado que as simulações com RANS conduziram a desvios na geração dos vórtices, o que induziu a predição subestimada da perda de carga e troca térmica neste problema.

Caciolo et al. [10] realizaram um estudo experimental e numérico do escoamento turbulento em um ambiente a ser ventilado por convecção natural. Os resultados desse estudo mostraram que a LES possui um potencial para obter uma predição mais adequada do que RANS para os parâmetros estudados, e.g., velocidades locais, temperaturas nas regiões de abertura do domínio. Contudo, o custo computacional requerido para as simulações com LES é, ao menos, uma ordem de magnitude maior do que as necessárias com RANS. Para escoamentos externos, Bouris e Bergeles [11] apresentaram um estudo em escoamentos externos sobre corpos rombudos comparando resultados experimentais e numéricos obtidos com RANS e LES. Neste estudo foi verificado que parâmetros médios como os coeficientes de arrasto e sustentação foram razoavelmente preditos com ambas abordagens. Contudo, o uso do modelo $k-\varepsilon$ conduziu a predições com grandes desvios nos campos locais de velocidade, principalmente na região de desprendimento de vórtices.

Uma motivação adicional para o presente estudo é auxiliar na compreensão da validade do emprego da modelagem clássica (RANS) para a simulação de escoamentos não-reativos com mecanismos combinados de transferência de calor por convecção e radiação térmica em meios participantes. As Interações Turbulência-Radiação (TRI - do inglês: Turbulence Radiation 
Interactions) têm sido investigadas principalmente no âmbito da modelagem clássica da turbulência, onde decomposições de Reynolds ou Favre são aplicadas as equações governantes e todas as flutuações e quantidades médias devem ser modeladas [12-14]. Com exceção do trabalho de Dos Santos et al. [8] que observaram que os efeitos de TRI são importantes para escoamentos não-reativos em meios participantes opticamente espessos, tem se afirmado na literatura que os efeitos de TRI podem ser negligenciados para escoamentos não reativos $[12,15]$. Para meios opticamente finos, todos os estudos têm observado que os efeitos de TRI podem ser negligenciados. Contudo, não se tem discutido sobre o emprego de diferentes modelos de fechamento para a predição dos campos fluido dinâmico, térmico e radiante nesse tipo de problema. Em outras palavras, a aplicação de RANS para o estudo de escoamentos turbulentos não-reativos com transferência de calor por convecção e radiação térmica em meios participantes só será viável se a predição dos campos médios e estatísticos de temperatura forem satisfatórios a ponto de não afetar os fluxos convectivos e radiantes entre o escoamento e as superfícies em consideração.

No presente trabalho é avaliado o emprego da Modelagem Clássica da Turbulência para a simulação de um escoamento turbulento de base cisalhante livre com convecção forçada em uma cavidade cilíndrica por meios da comparação com os campos preditos com LES. Nesse estudo é realizada uma comparação entre os campos médios e estatísticos de velocidades e temperaturas, bem como, os fluxos convectivos temporais médios nas superfícies da cavidade obtidos com LES e RANS. Foi considerado um escoamento com números de Reynolds e Prandtl de $\operatorname{Re}_{\mathrm{D}}=22000$ e $\operatorname{Pr}=0.71$. Além disso, considerou-se na entrada do jato na cavidade uma elevada intensidade de turbulência $(\mathrm{IT}=20 \%)$ que imita um caso extremo de escoamento não-reativo. As simulações do presente trabalho foram realizadas com o software de Dinâmica dos Fluidos Computacional FLUENT ${ }^{\circledR}$ [16], que é baseado no método de volumes finitos para as equações de conservação de massa, quantidade de movimento e energia [17,18]. Para a abordagem da turbulência emprega-se o modelo submalha dinâmico de Smagorinsky (DSSGS) $[19,20]$ para as simulações com LES e o modelo padrão $k-\varepsilon[6,21]$ para as simulações com RANS. O último modelo de fechamento foi selecionado com base na sua elevada aplicabilidade nas simulações de trabalhos da literatura [e.g. 13,14].

\section{MODELAGEM MATEMÁTICA}

\subsection{Simulação de Grandes Escalas (LES)}

A modelagem do escoamento transiente, incompressível com transferência de calor por convecção forçada é baseada na solução das equações de conservação (além das condições de contorno e iniciais). Na abordagem LES, as equações de conservação de massa, quantidade de movimento e energia são espacialmente filtradas com um filtro do tipo caixa [22]. Essas equações podem ser escritas por [4,5]:

$$
\begin{array}{ll}
\frac{\partial \bar{v}_{i}}{\partial x_{i}}=0 & (i=1,2 \text { e } 3) \text { em } t \times \Omega \\
\frac{\partial \bar{v}_{i}}{\partial t}+\frac{\partial\left(\bar{v}_{i} \bar{v}_{j}\right)}{\partial x_{j}}=-\frac{1}{\bar{\rho}} \frac{\partial \bar{p}}{\partial x_{j}} \delta_{i j}+\frac{\partial}{\partial x_{j}}\left\{v\left(\frac{\partial \bar{v}_{i}}{\partial x_{j}}+\frac{\partial \bar{v}_{j}}{\partial x_{i}}\right)-\tau_{i j}\right\} & (i, j=1,2 \text { e } 3) \text { em } t \times \Omega \\
\frac{\partial \bar{T}}{\partial t}+\frac{\partial}{\partial x_{j}}\left(\bar{v}_{j} \bar{T}\right)=\frac{\partial}{\partial x_{j}}\left\{\alpha \frac{\partial \bar{T}}{\partial x_{j}}-q_{j}\right\} & (i, j=1,2 \text { e } 3) \text { em } t \times \Omega
\end{array}
$$

onde () representa as grandes escalas (filtradas), $\rho$ é a massa específica do fluido $\left(\mathrm{kg} / \mathrm{m}^{3}\right), v$ é a viscosidade cinemática do fluido $\left(\mathrm{m}^{2} / \mathrm{s}\right) ; v_{\mathrm{i}}$ é a velocidade na direção $i, i=1,2$ e $3(\mathrm{~m} / \mathrm{s}) ; x_{\mathrm{i}}$ corresponde a coordenada espacial, $i=1,2$ e $3(\mathrm{~m}), p$ é a pressão $\left(\mathrm{N} / \mathrm{m}^{2}\right) ; T$ é a temperatura $(\mathrm{K})$; $\delta_{i j}$ é o delta de Kronecker, $\Omega$ é o domínio espacial (m) e $t$ é o tempo (s). Os termos $\tau_{i j}$ e $q_{\mathrm{j}}$ que surgem no processo de filtragem das equações de conservação de quantidade de movimento e energia, respectivamente, precisam ser modelados e são escritos por: 


$$
\begin{aligned}
\tau_{i j} & =\overline{v_{i} v_{j}}-\bar{v}_{i} \bar{v}_{j} \\
q_{j} & =\overline{v_{j} T}-\bar{v}_{j} \bar{T}
\end{aligned}
$$

O modelo submalha dinâmico de Smagorinsky é baseado na hipótese de Boussinesq [4]. Para um escoamento incompressível, o tensor de Reynolds submalha pode ser escrito por:

$$
\tau_{i j}=v_{s g s}\left(\frac{\partial \bar{v}_{i}}{\partial x_{j}}+\frac{\partial \bar{v}_{j}}{\partial x_{i}}\right)-\frac{2}{3} k \delta_{i j}
$$

onde $v_{\mathrm{sgg}}$ é a viscosidade submalha $\left(\mathrm{m}^{2} / \mathrm{s}\right)$ e $k$ é a energia cinética da turbulência $\left(\mathrm{m}^{2} / \mathrm{s}^{2}\right)$. O fluxo turbulento submalha é obtido analogamente ao tensor de Reynolds submalha e pode ser dado por $[4,5]$ :

$$
q_{j}=\alpha_{s g s} \frac{\partial \bar{T}}{\partial x_{j}}
$$

onde $\alpha_{s g s}$ é a difusividade térmica submalha $\left(\mathrm{m}^{2} / \mathrm{s}\right)$. Nesse modelo, as constantes de Smagorinsky, $C(x, t)$, e o número de Prandtl submalha, $\operatorname{Pr}_{\mathrm{sgs}}(x, t)$, são dinamicamente computados baseados na abordagem proposta por Germano et al. [19] e modificada por Lilly [20]. Essa modelagem é baseada no uso de dois filtros espaciais com diferentes comprimentos, providenciando informações sobre a transferência de energia das escalas resolvidas para as escalas não resolvidas do escoamento [4].

\subsection{Modelagem Clássica da Turbulência (RANS)}

As equações de conservação de massa, quantidade de movimento e energia médias no tempo são as mesmas apresentadas acima, Equações (1) - (3). Entretanto, para as simulações com RANS, () representa as variáveis médias no tempo. Por brevidade, essas equações não serão reapresentadas aqui.

Para a modelagem da turbulência é empregado o modelo a duas equações diferenciais $k-\varepsilon$ [6,21]. De acordo com esse modelo é requerida a solução de duas equações adicionais para a energia cinética da turbulência $(k)$ e sua taxa de dissipação $(\varepsilon)$ que são dadas por:

$$
\begin{aligned}
& \frac{\partial k}{\partial t}+\bar{v}_{j} \frac{\partial k}{\partial x_{j}}=\tau_{i j} \frac{\partial \bar{v}_{i}}{\partial x_{j}}+\frac{\partial}{\partial x_{j}}\left[\left(v+\frac{v_{t}}{\sigma_{k}}\right) \frac{\partial k}{\partial x_{j}}\right]-\varepsilon \\
& \frac{\partial \varepsilon}{\partial t}+\bar{v}_{j} \frac{\partial \varepsilon}{\partial x_{j}}=\frac{\partial}{\partial x_{j}}\left[\left(v+\frac{v_{t}}{\sigma_{\varepsilon}}\right) \frac{\partial \varepsilon}{\partial x_{j}}\right]+C_{\varepsilon 1} \frac{\varepsilon}{k} \tau_{i j} \frac{\partial \bar{v}_{i}}{\partial x_{j}}-C_{\varepsilon 2} \frac{\varepsilon^{2}}{k}
\end{aligned}
$$

onde a viscosidade turbulenta $\left(v_{t}\right)$ e a difusividade turbulenta $\left(\alpha_{t}\right)$ são dadas por:

$$
\begin{aligned}
& v_{t}=C_{\mu} \frac{k^{2}}{\varepsilon} \\
& \alpha_{t}=\frac{v_{t}}{\operatorname{Pr}_{t}}
\end{aligned}
$$

As constantes empregadas para resolver as equações de transporte adicionais, Equações (8) (11), são apresentadas na Tabela 1. 
Tabela 1: Constantes empregadas no modelo $k-\varepsilon$, equações $(8-11)$.

\begin{tabular}{cccccc}
\hline$C_{\mu}$ & $C_{\varepsilon 1}$ & $C_{\varepsilon 2}$ & $\sigma_{k}$ & $\sigma_{\varepsilon}$ & $\operatorname{Pr}_{t}$ \\
\hline 0.09 & 1.44 & 1.92 & 1.0 & 1.3 & 1.0 \\
\hline
\end{tabular}

\section{PROCEDIMENTOS NUMÉRICOS}

As equações de conservação que modelam o problema, Equações (1) - (3) são resolvidas usando o método de volumes finitos (FVM), mais precisamente empregando o software FLUENT [16]. O solver é baseado na pressão e todas simulações realizadas com LES empregaram o esquema de advecção de segunda ordem bounded central differencing, enquanto para as simulações com RANS foi empregado o esquema upwind de $2^{\mathrm{a}}$ ordem. O esquema empregado para o LES consiste de uma mistura de dois esquemas de advecção: diferenças finitas centrais para regiões onde o escoamento é difusivo dominante e upwind de $2^{\mathrm{a}}$ ordem em regiões onde a advecção é dominante [23]. O acoplamento pressão velocidade é realizado através do método SIMPLE. Maiores detalhes sobre o FVM podem ser encontrados em $[17,18]$.

As simulações numéricas foram realizadas em um computador com 6 processadores dualcore Intel i7 com $3.6 \mathrm{GHz}$ clock com uma memória instalada de 24 GB. Para a paralelização é empregada a biblioteca de passagem de mensagens (MPI - do inglês: Message Passing Interface). $\mathrm{O}$ tempo de processamento para as simulações com LES e RANS foram aproximadamente de $1.30 \times 10^{6} \mathrm{~s}$ e $9.30 \times 10^{4} \mathrm{~s}$, respectivamente. As simulações foram consideradas convergidas quando os resíduos para massa, velocidades e energia entre duas iterações consecutivas foram menores do que $10^{-4}, 10^{-6}$ e $10^{-8}$, respectivamente. Além disso, fatores de sub-relaxação de 0.7 foram impostos nas equações de conservação.

Com relação à avaliação das metodologias empregadas neste estudo, as mesmas foram validadas anteriormente no estudo de Dos Santos et al. [8] para a simulação de canais. Neste estudo específico os campos de temperaturas médios e estatísticos foram comparados com resultados obtidos por Kawamura et al. [24] empregando Simulação Numérica Direta (DNS). Para a simulação de escoamentos externos, os resultados para os coeficientes de arrasto, número de Strouhal, campos médios de velocidade e a energia cinética da turbulência foram comparados aos obtidos experimentalmente por Lyn et al. [25] e numericamente por Bouris e Bergeles [11]. Os resultados foram satisfatoriamente concordantes. Por simplicidade, os mesmos não serão apresentados neste trabalho.

\section{RESULTADOS E DISCUSSÃO}

A Figura 1 ilustra o domínio computacional do escoamento na cavidade cilíndrica estudado aqui. O domínio possui as seguintes dimensões: $R=D / 2=5.0 \times 10^{-2} \mathrm{~m}, R_{1}=12.5 \times 10^{-2} \mathrm{~m}, R_{2}=$ $25.0 \times 10^{-2} \mathrm{~m}$ and $L=1.7 \mathrm{~m}$. O princípio da similaridade é empregado para determinar as propriedades termofísicas do escoamento que possui como parâmetros fixos os números de Reynolds e Prandtl $\left(\operatorname{Re}_{\mathrm{D}}=22000\right.$ e $\left.\operatorname{Pr}=0.71\right)$. As condições de contorno impostas nas presentes simulações também são apresentadas na Figura 1. O escoamento é gerado pela imposição de um perfil de velocidades na entrada da cavidade cilíndrica com $v_{\text {in }}=100 \mathrm{~m} / \mathrm{s}$ e uma intensidade de turbulência de $20 \%$. Na mesma superfície, é imposta uma temperatura $T_{\text {in }}=2000 \mathrm{~K}$. Na saída da cavidade cilíndrica é imposta uma condição de tensão e fluxo de calor nulo. Para as demais superfícies, o campo de velocidades possui a condição de não deslizamento e impermeabilidade $\left(v_{1}=v_{2}=v_{3}=0 \mathrm{~m} / \mathrm{s}\right)$ e para o campo térmico é imposta uma temperatura fria constante $\left(T_{\mathrm{S}}=\right.$ $400 \mathrm{~K})$.

Para esse caso, o domínio foi discretizado com $50 \times 50 \times 65$ volumes nas direções $r, \theta$ e $z$ para ambas as simulações (LES e RANS). A malha é refinada na direção radial próximo as superfícies laterais (com 10 células em $y^{+}=5.0$ ) e na região do jato (20 células com uma razão de aspecto de $A R=1.05$ ). Nas direções $\theta$ e $z$, a malha foi considerada uniforme. Uma vez que não existe independência de malha nas simulações com LES, visto que o modelo depende da discretização espacial, o parâmetro empregado para verificar se a malha é adequada é o número de corte $\left(k_{\mathrm{c}}=\pi / \Delta x\right)$. São considerados valores adequados na literatura valores na faixa de $k_{\mathrm{c}}=$ 
$500 \mathrm{~m}^{-1}$ [7]. A malha empregada neste trabalho possui um refinamento ainda maior, sendo considerada um número de onda de corte de $k_{\mathrm{c}}=800 \mathrm{~m}^{-1}$. Uma vez que o refinamento requerido nas simulações com LES é maior do que as requeridas nas simulações com RANS, a malha empregada aqui também é adequada para as simulações com RANS. Vale destacar que, provavelmente um estudo de independência de malha nas simulações com RANS tivessem conduzido aos mesmos resultados com uma malha mais grosseira, economizando tempo de processamento. Considerando a discretização temporal, para ambas simulações foi empregado um passo de tempo $\Delta t=3.0 \times 10^{-5} \mathrm{~s}$. As simulações são realizadas até o tempo final de $t_{\mathrm{f}}=2.0 \mathrm{~s}$ e o intervalo de tempo $1.0 \leq t \leq 2.0 \mathrm{~s}$ foi utilizado para a determinação dos parâmetros médios $\mathrm{e}$ estatísticos dos campos de velocidades e temperaturas.

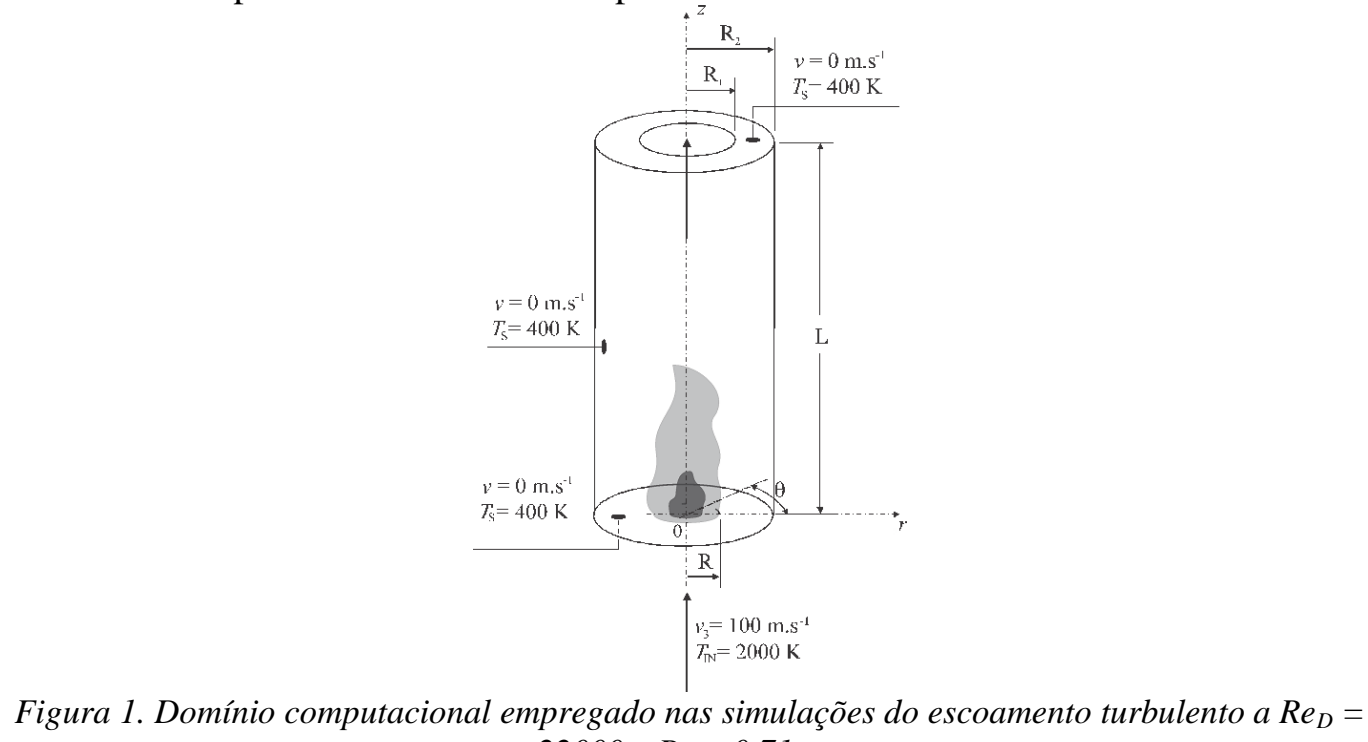
22000 e $\operatorname{Pr}=0.71$.

A Figura 2 apresenta os campos médios e estatísticos de velocidade obtidos com LES e RANS. As Figuras 2(a) e 2(b) mostram um comparativo dos campos médios de velocidades obtidos com LES e RANS, respectivamente. Os campos mostram que as predições com LES conduzem a uma maior penetração do jato na cavidade cilíndrica, enquanto na simulação com RANS há um maior espalhamento do jato na direção radial. Nas Figuras 2(c) e 2(d) são apresentados os campos com as velocidades RMS (do inglês - Root Mean Square) obtidas com LES e RANS. Da mesma forma que nos campos médios, há uma diferença entre as regiões de maior intensidade dos campos estatísticos ocorrem. Na simulação com LES as maiores intensidades de velocidade RMS ocorrem na região de saída da cavidade cilíndrica, enquanto na simulação com RANS esse efeito ocorre na região central da cavidade.

A Figura 3 apresenta os campos médios e estatísticos de temperatura obtidos com LES e RANS. As Figuras 3(a) e 3(b) mostram os campos médios de temperaturas obtidos com LES e RANS, respectivamente. Os campos mostram que a região central do jato possui uma maior penetração na cavidade para a simulação com LES e um maior espalhamento nas simulações com RANS, similarmente ao observado nos campos de velocidade. Com relação aos campos de temperatura RMS obtidos com LES e RANS, Figuras 3(c) e 3(d), respectivamente, são observadas diferenças nas regiões de maior magnitude de temperatura RMS e menor magnitude. Na simulação com LES se observam três regiões de maior magnitude (nas laterais do jato e na saída da cavidade) enquanto que na simulação com RANS há uma região de máxima magnitude na região de saída da cavidade.

Com o objetivo de avaliar as diferenças locais dos campos de velocidade e temperaturas obtidas com LES e RANS de forma quantitativa, três linhas de monitoramento foram inseridas ao longo do domínio da cavidade, mais precisamente nas posições $z=0.2 \mathrm{~m}, z=0.4 \mathrm{~m}$ e $z=1.2 \mathrm{~m}$, conforme ilustram as Figuras $4-6$, respectivamente. 

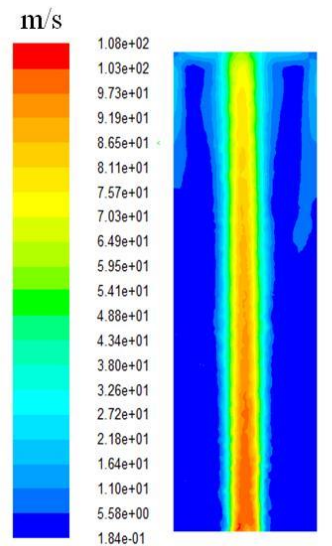

a)

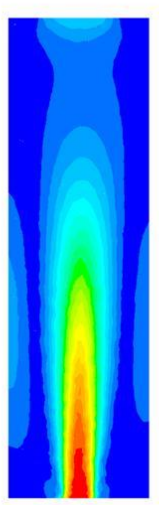

b)

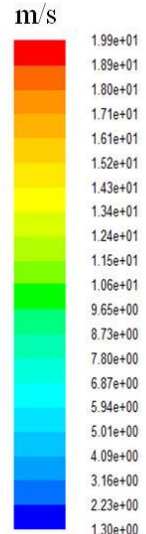

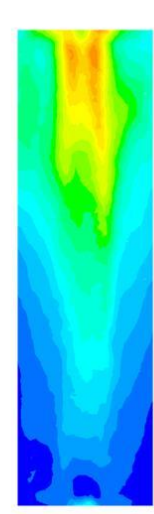

c) $\mathrm{m} / \mathrm{s}$

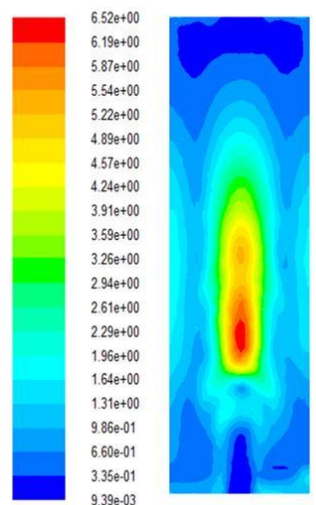

d)

Figura 2: Campos de velocidades para escoamento com convecção forçada a $\operatorname{Re}_{D}=22000$ e $\operatorname{Pr}=0.71$ : campos médios a) LES, b) RANS $k-\varepsilon$, velocidade RMS: c) LES, d) RANS $k-\varepsilon$.

$\mathrm{K}$

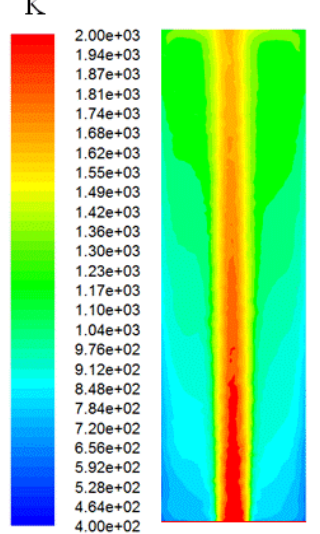

a)

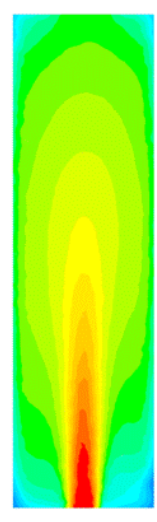

b)

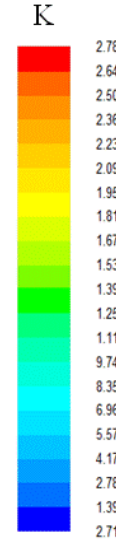

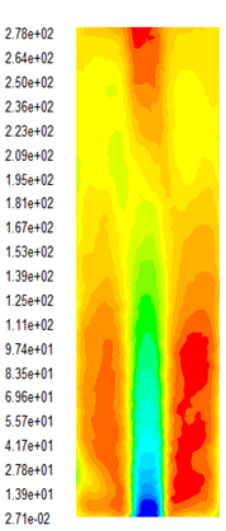

c)

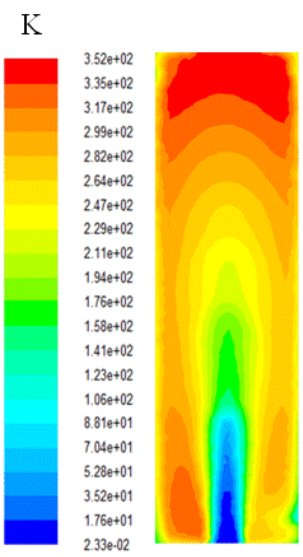

d)

Figura 3: Campos de temperaturas para escoamento com convecção forçada a $\operatorname{Re}_{D}=22000$ e $\operatorname{Pr}=0.71$ : campos médios a) LES, b) RANS $k-\varepsilon$, temperatura RMS c) LES, d) RANS $k-\varepsilon$.

A Figura 4(a) mostra os perfis de velocidades médios e RMS obtidos com LES e RANS quando o escoamento chega ao regime permanente. Em geral, os perfis médios e estatísticos foram qualitativamente semelhantes. Os campos de velocidades médios foram concordantes. Por outro lado, a magnitude da velocidade RMS predita pela simulação com LES foi sensivelmente superior do que a obtida para a simulação com RANS. A magnitude do perfil de velocidade RMS predito com LES foi, em média, 5 vezes superior ao apresentado com RANS. Na Figura 4(b) são apresentados os perfis de temperaturas médios e RMS obtidos com LES e RANS. Em média, os perfis obtidos com o modelo RANS tiveram maior magnitude do que os preditos com LES. Essa diferença é bastante evidente para o perfil médio de temperaturas na região afastada da região central do jato. As predições com LES e RANS conduziram a diferenças nos campos médios e estatísticos de até $600 \mathrm{~K}$ e $80 \mathrm{~K}$. Essas diferenças podem ser significativas na predição dos fluxos convectivos para casos de convecção forçada e também podem levar a diferenças consideráveis na predição dos fluxos radiantes em estudos de escoamentos não-reativos em meios participantes com transferência de calor por convecção e radiação térmica, principalmente para meios opticamente espessos, onde os campos locais de temperatura apresentam maior influência no fluxo radiante das superfícies do domínio.

A Figura 5 ilustra os campos de velocidades e temperaturas médios e RMS para $z=0.4 \mathrm{~m}$. Da mesma forma que para $z=0.2 \mathrm{~m}$, foram observadas diferenças nas velocidades RMS (com uma magnitude maior na simulação com LES) e no campo de temperaturas médio, onde a magnitude é maior na região central com LES e na periferia com RANS. Para esta posição, foram observadas diferenças de até $403 \mathrm{~K}$ e $73 \mathrm{~K}$ nos campos médios e estatísticos de 
temperatura, respectivamente. Na Figura $6(z=1.2 \mathrm{~m})$ as diferenças dos campos médios de velocidades, Figura 6(a), e temperaturas, Figura 6(b), principalmente na região central, ficam ainda mais evidentes devido aos campos médios preditos com RANS já estarem amortecidos nessa região. Apesar disso, a magnitude da temperatura RMS na predição com RANS continuou sendo superiores as obtidas com LES.

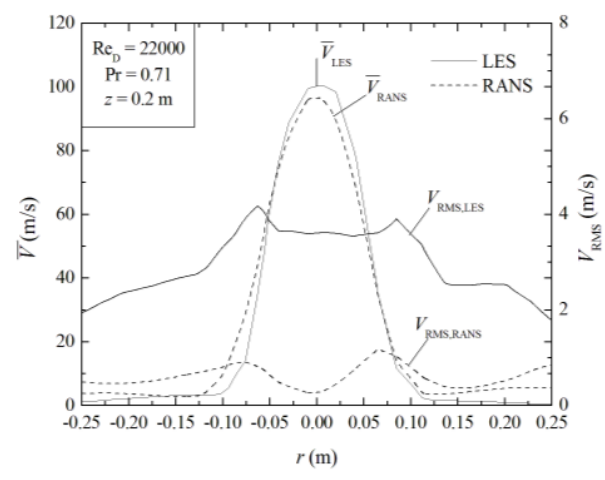

a)

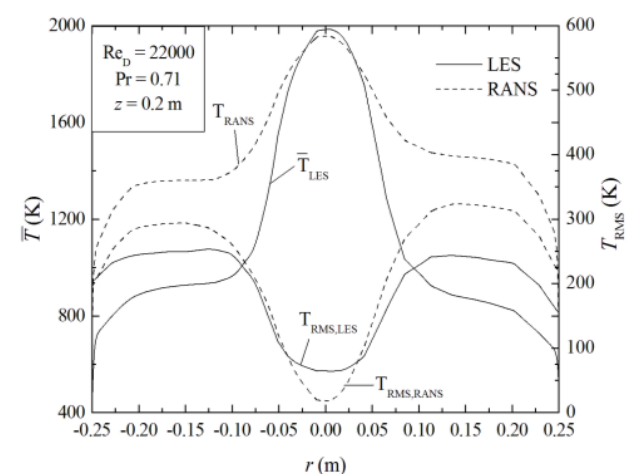

b)

Figura 4: Comparação entre perfis de velocidades e temperaturas obtidos com LES e RANS $k-\varepsilon$ em $z=$ $0.2 \mathrm{~m}:$ a) velocidade média e RMS, b) temperatura média e RMS.

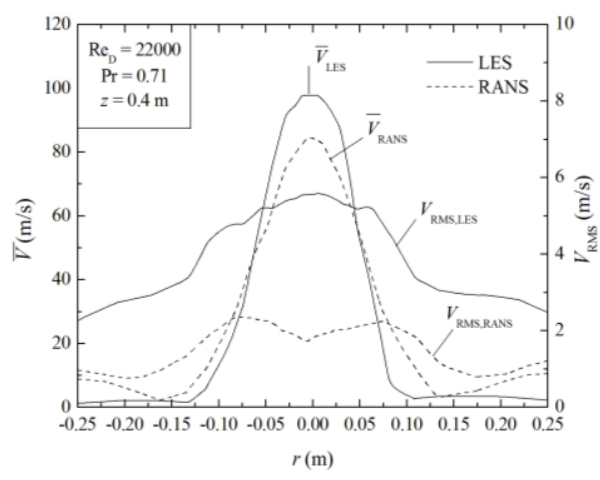

a)

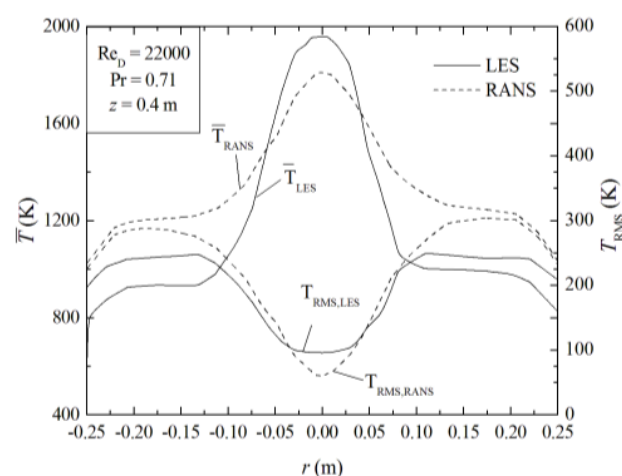

b)

Figura 5: Comparação entre perfis de velocidades e temperaturas obtidos com LES e RANS $k-\varepsilon$ em $z=$ $0.4 \mathrm{~m}$ : a) velocidade média e RMS, b) temperatura média e RMS.

A Figura 7 ilustra a comparação dos fluxos convectivos obtidos com LES e RANS na região da parede da cavidade cilíndrica. Pode ser observado que há uma grande defasagem entre a região onde ocorre o máximo fluxo para as simulações com RANS e LES. Em RANS o ponto de máximo é observado para $z=0.90 \mathrm{~m}$, enquanto na simulação com LES esse ponto se desloca para $z=1.63 \mathrm{~m}$. Esse comportamento reflete a diferença na predição de comprimento dos jatos obtidos com ambas metodologias. Também é observado que devido ao maior espalhamento do jato na predição com RANS, rapidamente o fluxo convectivo aumenta e permanece com uma elevada magnitude na faixa $0.5 \mathrm{~m} \leq z \leq 1.2 \mathrm{~m}$. Enquanto isso, na simulação com LES há um crescimento abrupto do fluxo a partir de $z=1.2 \mathrm{~m}$ até o seu máximo seguido de um rápido decréscimo, ou seja, o comportamento do fluxo em função da coordenada espacial é bastante diferente para ambas as predições.

\section{CONCLUSÃO}

O presente trabalho apresentou um estudo numérico comparando o emprego de LES e RANS com modelo $k-\varepsilon$ para a predição dos campos médios, estatísticos e fluxos convectivos em um escoamento turbulento com convecção forçada em cavidades cilíndricas a $\operatorname{Re}_{\mathrm{D}}=22000$ e $\operatorname{Pr}=$ 0.71. As equações de conservação foram resolvidas através do Método de Volumes Finitos 
(FVM).

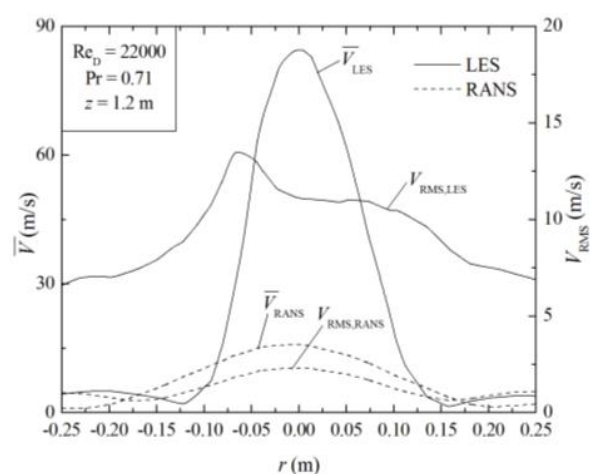

a)

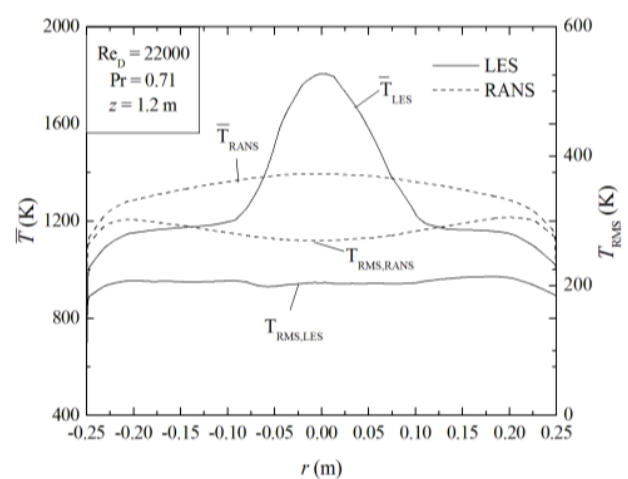

b)

Figura 6: Comparação entre perfis de velocidades e temperaturas obtidos com LES e RANS $k-\varepsilon$ em $z=$ $1.2 \mathrm{~m}$ : a) velocidade média e RMS, b) temperatura média e RMS.

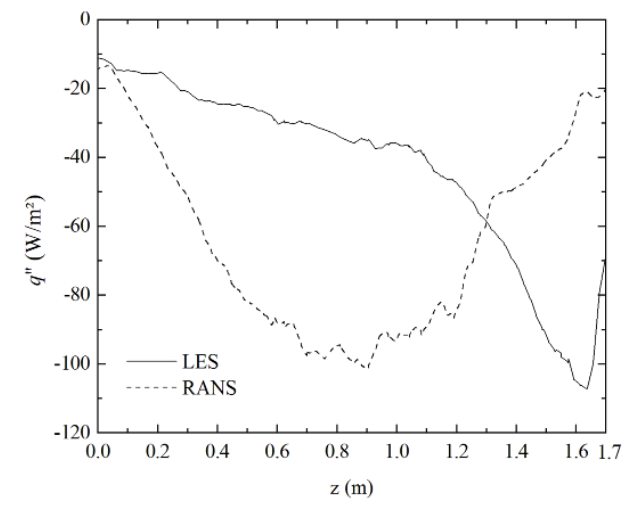

Figura 7: Comparação dos fluxos convectivos obtidos com LES e RANS $k-\varepsilon$.

Os resultados indicaram que os escoamentos preditos com LES e RANS apresentaram grandes diferenças, com as simulações realizadas em RANS subestimando o comprimento do jato na câmara cilíndrica. Esse comportamento é semelhante ao observado para a simulação de escoamentos externos sobre cilindros e corpos rombudos, e.g., [11].

Também foram observadas diferenças significativas nos campos médios e estatísticos de velocidades e, principalmente de temperaturas. Por exemplo, para $\mathrm{z}=0.2 \mathrm{~m}$ foram obtidas diferenças nas predições com LES e RANS de até $600 \mathrm{~K}$ e $80 \mathrm{~K}$ nos campos médios de temperatura e temperatura RMS, respectivamente. Essas diferenças foram determinantes para predições significativamente diferentes nos fluxos convectivos, tanto no comportamento qualitativo quanto na magnitude ao longo da superfície da cavidade. Essas diferenças também podem ser importantes em futuras predições de escoamentos com transferência de calor por convecção e radiação térmica em meios participantes.

Para continuação deste trabalho serão avaliados outros modelos clássicos de turbulência como $k-\omega$ e RSM e outras intensidades de turbulência para avaliar o efeito desse parâmetro sobre as diferenças nas predições entre as abordagens LES e RANS.

\section{AGRADECIMENTOS}

E. D. dos Santos, F. H. R. França, J. A. Souza, L. A. Isoldi e L. A. O. Rocha agradecem ao $\mathrm{CNPq}$ pelo suporte financeiro. 


\section{REFERÊNCIAS BIBLIOGRÁFICAS}

1. Pope SB. Turbulent flows. Cambridge: Cambridge University Press; 2008. 802 p.

2. Coelho PJ. Numerical simulation of the interaction between turbulence and radiation in reactive flows. Prog Energy Combust Sci. 2007 Jan;33(4):311-83, doi:10.1016/j.pecs.2006.11.002.

3. Kasagi N, Iida O. Progress in direct numerical simulation of turbulent heat transfer. In: Proceedings of the 5th ASME/JSME Joint Thermal Engineering Conference; 1999; San Diego, CA. Seattle; p. 117.

4. Lesieur M, Métais O, Comte P. Large-eddy simulations of turbulence. Cambridge: Cambridge University Press; 2005. 232 p.

5. Sagaut P. Large eddy simulation for incompressible flows, an introduction. Berlin: Springer-Verlag Berlin Heidelberg; 2006. 558 p.

6. Wilcox DC. Turbulence modeling for CFD. La Canada: DCW Industries; 2002. 540 p.

7. Dos Santos ED, Piccoli GL, França FHR, Petry AP. Analysis of mixed convection in transient laminar and turbulent flows in driven cavities. Int J Heat Mass Transf. 2011 Jul;54(21-22):4585-95, doi:10.1016/j.ijheatmasstransfer.2011.06.019.

8. Dos Santos ED, Isoldi LA, Petry AP, França FHR. A numerical study of combined convective and radiative heat transfer in non-reactive turbulent channel flows with several optical thicknesses: a comparison between LES and RANS. J Braz Soc Mech Sci Eng. 2014 Aug;36(1):207-19, doi:10.1007/s40430-013-0075-1.

9. Colleoni A, Toutant A, Olalde G. Simulation of an innovative internal design of a plate solar receiver: Comparison between RANS and LES results. Sol Energy. 2014 May;105:732-41, doi:10.1016/j.solener.2014.04.017.

10. Caciolo M, Stabat P, Marchio D. Numerical simulation of single-sided ventilation using RANS and LES and comparison with full-scale experiments. Build Environ. 2012 Nov;50:202-13, doi:10.1016/j.buildenv.2011.10.017.

11. Bouris D, Bergeles G. 2D LES of vortex shedding from a square cylinder. J Wind Eng Ind Aerodyn. 1999 Mar;80(1-2):31-46, doi:10.1016/S0167-6105(98)00200-1.

12. Mazumder S, Modest MF. A probability density function approach to modeling turbulence-radiation interactions in nonluminous flames. Int $\mathbf{J}$ Heat Mass Transf. 1999 Mar;42(6):971-91, doi:10.1016/S0017-9310(98)00225-7.

13. Li G, Modest MF. Importance of turbulence-radiation interactions in turbulent diffusion jet flames. J Heat Transf. 2003 Oct;125(5):831-38, doi:10.1115/1.1597621.

14. Wang A, Modest MF, Haworth DC, Wang L. Monte Carlo simulation of radiative heat transfer and turbulence interactions in methane/air jet flames. J Quant Spectrosc Radiat Transf. 2008 Jan;109(2):269-79, doi:10.1016/j.jqsrt.2007.08.030.

15. Gupta A, Modest MF, Haworth DC. Large-eddy simulation of turbulence-radiation interactions in a turbulent planar channel flow. J Heat Transf. 2009 Apr;131(6):061704(8pages), doi:10.1115/1.3085875.

16. FLUENT. Documentation Manual - FLUENT 6.3.16. Ansys Inc.; 2007.

17. Patankar SV Numerical heat transfer and fluid flow. New York: McGraw Hill; 1980. 214 p.

18. Versteeg HK, Malalasekera W. An Introduction to computational fluid dynamics: the finite volume method. London: Pearson-Prentice Hall; 2007. 503 p.

19. Germano M, Piomelli U, Moin P, Cabot WH. A dynamic sub-grid-scale eddy viscosity model. Phys Fluids A. 1991 Mar;3:1760-5, http://dx.doi.org/10.1063/1.857955.

20. Lilly DK. A proposal modification of the Germano subgrid-scale closure method. Phys Fluids A. Nov 1992;4:633-5, http://dx.doi.org/10.1063/1.858280.

21. Launder BE, Spalding DB. Lectures in mathematical models of turbulence. London: Academic Press; 1972. 169 p.

22. Findikakis AN, Street RL. Mathematical description of turbulent flows. J Hydraul Div-ASCE. 1982 Aug;108(2):887-903.

23. Zhu J, Rodi W. A low dispersion and bounded convection scheme. Comput Methods Appl Mech Eng. 1991 Nov;92(1):225-32, doi:10.1016/0045-7825(91)90199-G.

24. Kawamura H, Abe H, Shingai K. DNS of turbulence and heat transport in a channel flow with Reynolds and Prandtl numbers and boundary conditions. In: 3rd International Symposium on Turbulence, Heat and Mass Transfer; 2000; Nagoya.

25. Lyn D, Elinav S, Rodi W. Park J. A laser doppler velocimetry study of ensemble-averaged characteristics of the turbulent near wake of a square cylinder. J Fluid Mech. 1995 Dec;304:285319, doi:http://dx.doi.org/10.1017/S0022112095004435. 